\title{
Single Abscess as the First Manifestation of Breast ALK-Positive Anaplastic Large Cell Lymphoma in Pregnancy Without Breast Implants: A Case Report
}

\author{
Da-Ping Song ${ }^{1 *}$, Wen-Yan Zhang ${ }^{2}$ \\ 1The Third Hospital of Mianyang, Sichuan Mental Health Center, Mianyang 621000, China \\ 2West China Hospital Sichuan University, Chengdu 610044, China \\ *Corresponding author: Da-Ping Song, 809906@qq.com
}

Copyright: ( $) 2022$ Author(s). This is an open-access article distributed under the terms of the Creative Commons Attribution License (CC BY 4.0), permitting distribution and reproduction in any medium, provided the original work is cited.

\begin{abstract}
Primary lymphoma involving the breast accounts for only $0.04-0.5 \%$ of malignant breast tumors, constituting $1-$ $2 \%$ of all non-Hodgkin lymphomas (NHL). Here, we report a case of breast ALK-positive ALCL arising in a 21-year-old woman in pregnancy with single abscess as the first clinical manifestation.
\end{abstract}

Keywords: ALK-positive anaplastic large cell lymphoma in pregnancy; Breast implants

Online publication: January 20, 2022

\section{Introduction}

Primary lymphoma involving the breast accounts for only $0.04-0.5 \%$ of malignant breast tumors, constituting 1-2\% of all non-Hodgkin lymphomas (NHL) ${ }^{[1]}$. Most patients were female with unilateral breast, rarely bilateral breast, whereas the vast majority of primary breast lymphomas (95\%) are of B-cell origin with diffuse large B-cell lymphoma (DLBCL) or mucosa-associated lymphoid tissue lymphomas (MALT lymphoma) ${ }^{[2]}$. Of the $<10 \%$ that are of T lineage, most are PTCL-NOS ${ }^{[3]}$. Primary anaplastic large cell lymphoma (ALCL) of the breast is very rare. Breast implant-associated ALCL (BIA-ALCL) are predominantly T-cell neoplasms ALCL ${ }^{[4]}$, with few cases of B-cell lymphomas described in association with breast implants ${ }^{[5]}$.

\section{Case presentation}

A 21-years-old female patient (gravida 1, para 0 ) in the $28^{\text {th }}$ week of gestation was admitted to the hospital with left breast mass on January 8, 2016. The patient denied any history of fever, night sweats or weight loss. A 10-cm mass-like abscess in the left breast was evident in physical examination with obscure bound, pain with touch, red and swelling in the area. Two drainage incisions were made in upper inner and lateral quadrant of left breast, without nipple discharge or crater nipple (Figure 1a). The right breast was normal. Diagnosis was delayed because many doctors thought it was abscess of breast, with incision and drainage. The mass continued to grow, and the patient was then referred to us for therapeutic management. B-mode ultrasonography showed fluid sonolucent area in the left breast. Pathologic diagnosis indicates that the neoplastic cells are large, epithelioid, and pleomorphic, with abundant cytoplasm, vesicular irregular nuclei, 
and frequent mitoses. "Hallmark" cells could be found (Figure 3). The cells typically showed strong and diffuse immunoreactivity for CD30 and ALKT, T-cell markers (CD3), cytotoxic-associated antigens, and epithelial membrane antigen (EMA), but negative expression for CD20, CD79a and Pax-5. Molecular genetic analyses have demonstrated T-cell receptor gene rearrangements. Histology, immunohistochemistry, and T-cell gene rearrangement studies were supportive of a CD30- and ALK-1positive anaplastic large cell lymphoma. Patient was disposed of the induction labor in the $32^{\text {nd }}$ week of gestation. After two days, computed tomography (CT) imaging showed multiple system lesions with mass in the left breast, an enlarged numerous bilateral cervical and left axillary lymph nodes, multiple nodules in the left lung, and single nodule in the left kidney (Figure 2a). Biopsy of bone marrow and flow cytometry were performed and no involvement was noted. The patient was treated with 6 cycles of cyclophosphamide, doxorubicin, vincristine, and prednisone combination chemotherapy (CHOP). Three cycles later, repeated CT imaging showed reduced mass of left breast and bilateral cervical and left axillary lymph nodes, as well as loss of nodules in the left lung and nodule in the left kidney. Six cycles later, the left breast was normal (Figure 1b). Repeated CT imaging showed that the patient had no bilateral cervical or axillary lymphadenopathy, no abscess in the left breast (Figure $\mathbf{2 b}$ ). In a follow-up period of 3 months, there was no evidence of disease recurrence.

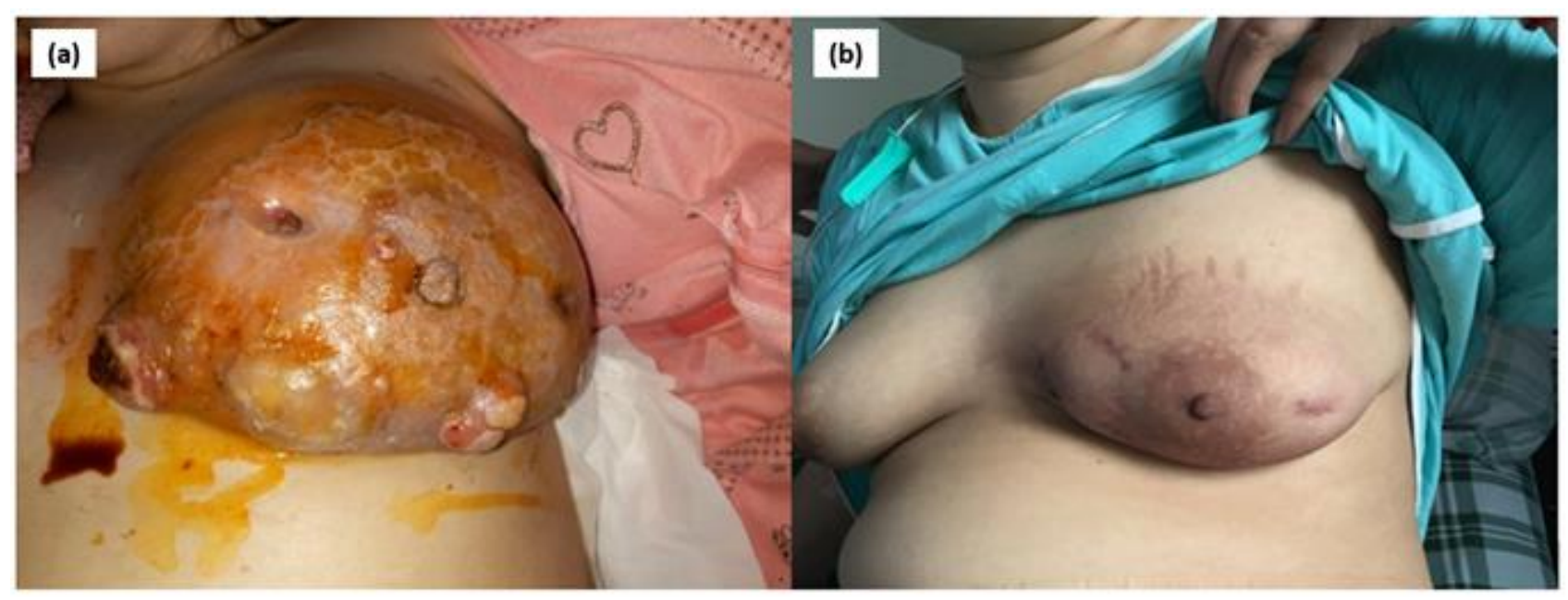

Figure 1. (a) Prior to treatment. (b) 6 cycles of cyclophosphamide later.
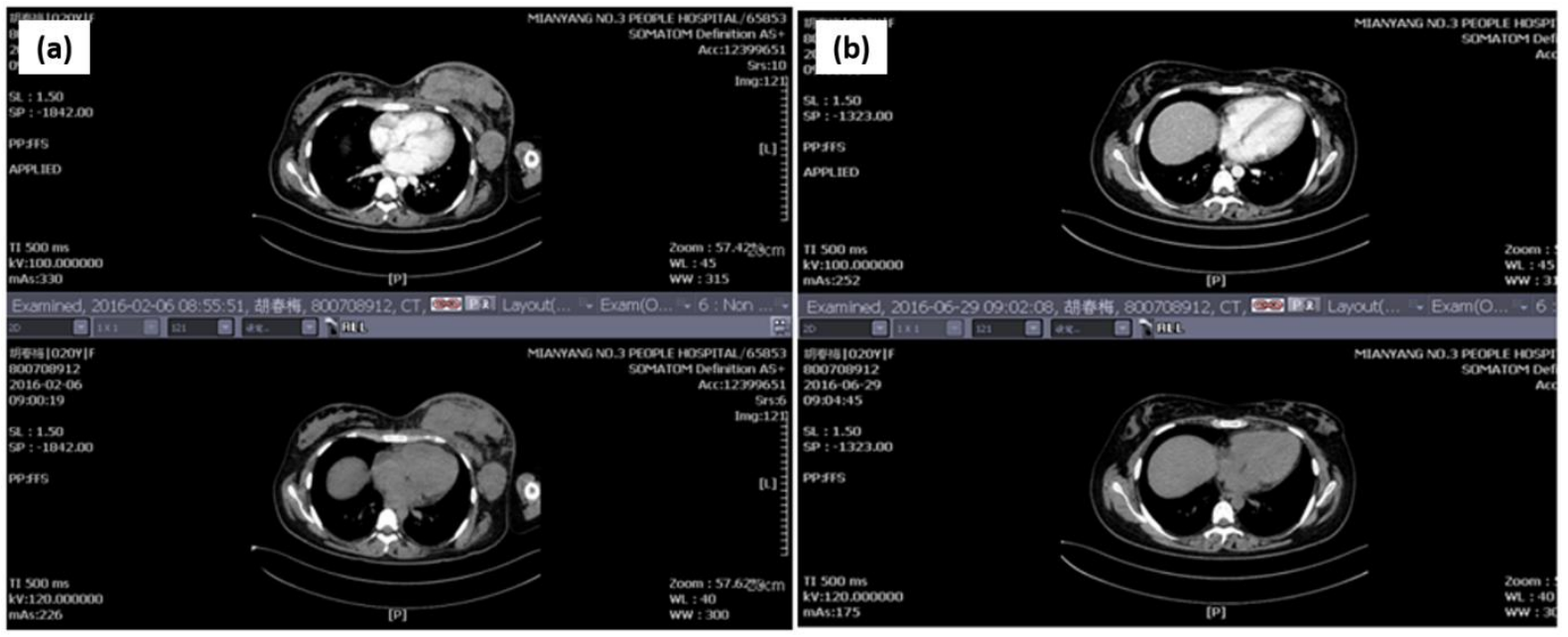

Figure 2. (a) Prior to treatment. (b) 6 cycles cyclophosphamide of later. 


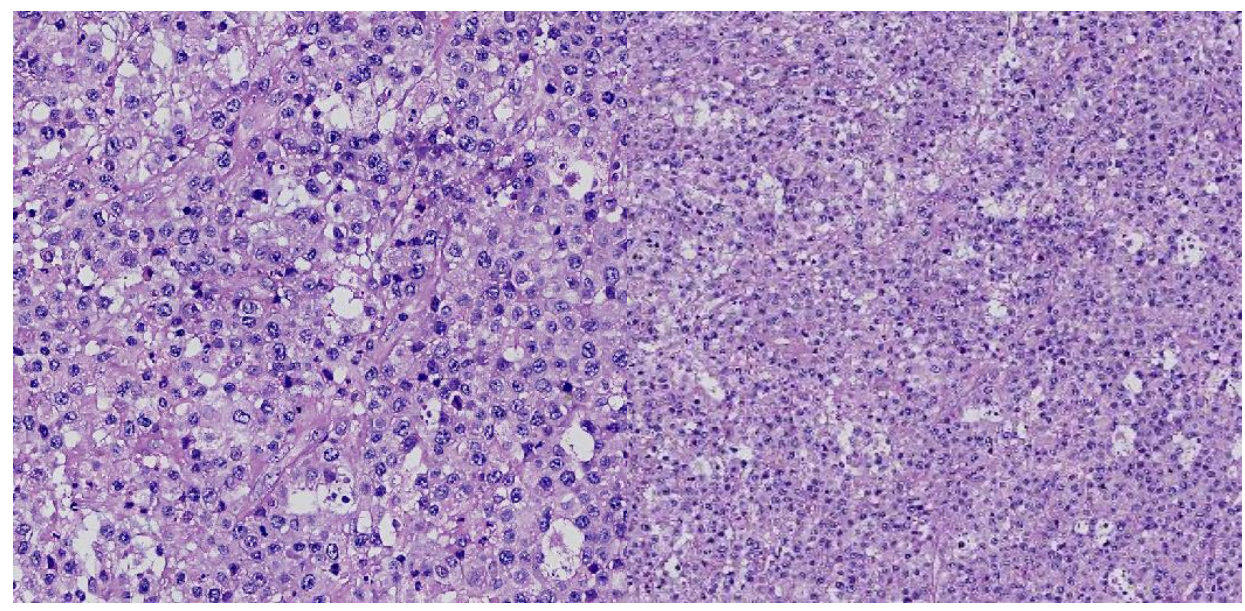

Figure 3. Histopathological image of breast.

\section{Discussion}

NSCHL is the most prevalent subtype of Hodgkin's lymphoma (HL) during pregnancy. Bcell lymphoma and aggressive subtypes are most common for NHL during pregnancy. The outcomes of NHL are inferior to those of HL during pregnancy ${ }^{[6]}$. Primary breast ALCL is rare but is more commonly seen in patients with implants. BIA-ALCL is not a disease of the breast parenchyma but instead, is a disease of the fibrous capsule surrounding the implant. The patients usually present with an effusion around the implant and, rarely, with a solid mass ${ }^{[7]}$. The clinical findings were seroma (67.33\%), nodes (13.8\%), mass $(22.1 \%)$, other $(11.7 \%)$ and unknown (32\%). Breast abscess is very rare. In $97 \%$ of the cases, ALK was negative and $3 \%$ were positive ${ }^{[8]}$. The most common marker was CD30. Almost 200 women worldwide have been diagnosed with BIA-ALCL. It is postulated that chronic inflammation via bacterial infection may be an etiological factor ${ }^{[9]}$. ALCL, a rare form of T-cell NHL, is classified as systemic, secondary, or primary cutaneous. Systemic ALCL often follow an aggressive clinical course and is usually driven by ALK expression ${ }^{[10]}$. In most BIA-ALCL cases, it was less aggressive, which is frequently ALK-negative and follows an indolent course ${ }^{[11]}$. Many specialists refer BIA-ALCL to primary cutaneous ALCL ${ }^{[11]}$. The disease was confined to the capsule. Few patients developed aggressive disease which was extracapsular and showed bad prognosis ${ }^{[8]}$. However, BIA-ALCL has many unique clinical and pathological features, suggesting that it is a separate disease entity ${ }^{[12]}$. In 2015, a multidisciplinary panel of 12 experts agreed that patients with BIA-ALCL should undergo affected implant removal and total capsulectomy, and disagreed with statements that chemotherapy and radiation therapy should be given to all patients ${ }^{[13]}$. Other analysis suggests that patients with BIA-ALCL that are confined to the capsular fibrous tissue generally respond well with surgical excision alone, whereas those with a tumor mass may have a more aggressive course and poor prognosis, requiring chemotherapy and/or radiation therapy ${ }^{[14]}$. Chemotherapy and/or radiation therapy should be given to all patients with systemic ALCL. A recent review of the literature has shown that chemotherapy regimens, such as regimen containing doxorubicin, bleomycin, vinblastine and dacarbazine as well as regimen containing cyclophosphamide, doxorubicin, vincristine and prednisone, are safe during the second and third trimesters in pregnancy with no delayed fetal adverse events ${ }^{[15]}$.

We report a very rare case of breast ALK-positive ALCL arising in pregnancy without history of breast implant. Diagnostic was delayed because abscess of clinical manifestation, owing to the rare possibility clinical manifestation of ALCL.

\section{Disclosure statement}

The authors declare no conflict of interest. 


\section{References}

[1] Talwalkar SS, Miranda RN, Valbuena JR, et al., 2008, Lymphomas Involving the Breast: A Study of 106 Cases Comparing Localized and Disseminated Neoplasms [J]. Am J Surg Pathology, 32(9): 12991309.

[2] Lv X, Yu C, Zhao S, et al., 2015, The Progress of Primary Breast Lymphoma. Modern Oncology, 8(23): 1149-1151.

[3] Roden AC, Macon WR, Keeney GL, et al., 2008, Seroma-Associated Primary Anaplastic Large Cell Lymphoma Adjacent to Breast Implants: An Indolent T Cell Lymphoproliferative Disorder. Mod Pathol, 21(4): 455-463.

[4] Lazzeri D, Agostini T, Bocci G, et al., 2011, ALK-Negative Anaplastic Large Cell Lymphoma Associated with Breast Implants: A New Clinical Entity. Clin Breast Cancer, 11: 283-296.

[5] Carty MJ, Pribaz JJ, Antin JH, et al., 2011, A Patient Death Attributable to Implant-Related Primary Anaplastic Large Cell Lymphoma of the Breast. Plast Reconstr Surg, 128: 112e-118e.

[6] Gui L, Shi Y, He X, et al., 2015, Clinicopathological Features and Outcomes: Analysis of 21 Cases of Lymphoma in Pregnancy. Zhonghua Yi Xue Za Zhi, 95(6): 425-429.

[7] Xu J, Wei S, 2014, Breast Implant-Associated Anaplastic Large Cell Lymphoma: Review of a Distinct Clinicopathologic Entity. Arch Pathol Lab Med, 138(6): 842-846.

[8] Ramos-Gallardo G, Cuenca-Pardo J, 2016, Breast Implant and Anaplastic Large Cell Lymphoma Meta-Analysis. J Invest Surg, 18: 1-10.

[9] Kadin ME, Deva A, 2016, Biomarkers Provide Clues to Early Events in the Pathogenesis of Breast Implant-Associated Anaplastic Large Cell Lymphoma. Aesthet Surg J, 36(7): 773-781.

[10] Lechner MG, Lade S, Liebertz DJ, et al., 2011, Breast Implant-Associated, ALK-Negative, T-cell, Anaplastic, Large-Cell Lymphoma: Establishment and Characterization of a Model Cell Line (TLBR1) for This Newly Emerging Clinical Entity. Cancer, 117: 1478-1489.

[11] Story SK, Schowalter MK, Geskin LJ, 2013, Breast Implant-Associated ALCL: A Unique Entity in the Spectrum of CD30+ Lymphoproliferative Disorders. Oncologist, 18: 301-307.

[12] George EV, Pharm J, Houston C, et al., 2013, Breast Implant-Associated ALK-Negative Anaplastic Large Cell Lymphoma:A Case Report and Discussion of Possible Pathogenesis. Int J Clin Exp Pathol, 6(8): 1631-1642.

[13] Kim B, Zachary S, Predmore BA, et al., 2015, Breast Implant-Associated Anaplastic Large Cell Lymphoma: Updated Results from a Structured Expert Consultation Process. Plast Reconstr Surg Glob Open, 3(1): e296.

[14] Miranda RN, Aladily TN, Prince HM, et al. 2014, Breast Implant-Associated Anaplastic Large-Cell Lymphoma: Long-Term Follow-Up of 60 Patients. J Clin Oncol, 32: 114-120.

[15] Pereg D, Koren G, Lishner M, 2007, The Treatment of Hodgkin's and non-Hodgkin's Lymphoma in Pregnancy. Haematologica, 92: 1230-1237. 